\title{
Réflexions sur la traduction de la littérature enfantine africaine : Véronique Tadjo et Michelle Tanon-Lora ${ }^{1}$
}

Ce travail voudrait proposer une réflexion sur la langue de trois textes africains de littérature pour la jeunesse, à partir des difficultés que l'on a rencontrées dans la phase de traduction en langue italienne qui a été l'objet de notre mémoire ${ }^{2}$. Deux de ces textes Mamy Wata et le monstre (MW) et Masque, raconte-moi $(M R)$ ont été écrits par la célèbre femme de lettres ivoirienne Véronique Tadjo, tandis que le troisième, La ceinture de madame Fourmi (LC) a été signé par sa compatriote Michelle Tanon-Lora, qui s'est consacrée à la littérature enfantine en 2009. La comparaison entre les deux auteurs révèle aussi bien la parole dense et plurivalente de la première, catalysée par un usage attentif d'images et de couleurs, que l'énergie et la vitalité qui marquent l'écriture de la seconde. Très différentes de par leur âge, leur formation, les œuvres publiées, elles sont pourtant toutes deux animées de la même foi en la force d'une éducation solide pour les nouvelles générations investies du pouvoir de changer la société. Voilà ce qui les a poussées à travailler dans la même direction après des carrières si différentes, dans un domaine aussi difficile que la littérature pour la jeunesse, surtout pour celui qui se trouve partagé entre les lecteurs occidentaux et son public national, que l'analphabétisme et la pauvreté maintient à l'état d'embryon ${ }^{3}$.

Le sens de responsabilité que les deux écrivains ressentent envers leur public les pousse à créer des œuvres de qualité, qui stimulent de façon appropriée des tranches d'âge si réceptives et influençables. Leur engagement découle de l'idée que la misère et l'ignorance se nourrissent l'une de l'autre, et que le travail avec les enfants est le seul moyen pour briser ce cercle vicieux. Bien qu'elles appartiennent à la littérature de jeunesse, les œuvres considérées visent un public plus vaste qu'elles cherchent à former. L'approche de la traduction impose donc de considérer toujours ce « double destinataire » auquel ces deux femmes s'adressent, afin de déterminer et de respecter la soi-disant «intention du texte » (Eco 16), et d'éviter tantôt des tentations de lyrisme, tantôt des hardiesses de pédagogie improvisée. Dans le cas de Michelle Tanon Lora (mais Véronique Tadjo a contribué elle aussi à maintes initiatives de ce type) le travail d'écriture s'ajoute à l'activité de tous les jours

\footnotetext{
${ }^{1}$ Cet article a été sélectionné par le comité éditorial de la revue parmi plusieurs contributions d'étudiants gradués.

${ }^{2}$ Toutes les traductions en langue italienne qui suivent dans le texte sont tirées de mon mémoire de maitrise intitulé Riflessioni sulla lingua di alcuni testi africani per l'infanzia et effectué sous la direction de Nataša Raschi.

3 Lire à ce propos l'article de Boucabar Boris Diop "Identité africaine et mondialisation ", publié dans Africultures. Il se concentre sur les rapports entre les écrivains africains et leur public international, en décrivant le bizarre jeu de miroirs qui fait que ce sont les écrivains africains qui vivent depuis des années à l'étranger et ne connaissent presque plus rien de l'Afrique qui sont les plus écoutés, parce qu'ils sont légitimés par les élites culturelles de l'Occident.
} 
avec les enfants des banlieues métropolitaines ${ }^{4}$ qui, parmi de nombreuses difficultés, sont conduits dans les rares bibliothèques disponibles, où ils peuvent entrer en contact avant tout avec l'objet-livre, encore si peu diffusé en Afrique ${ }^{5}$.

En outre, nos deux écrivains visent à la fois à préserver et à actualiser l'esprit de la littérature orale, leurs textes étant conçus non seulement pour être lus à haute voix, mais aussi dansés, chantés et "joués ${ }^{6}$ ». Elles essayent de créer une didactique nouvelle, qui fasse des enfants les sujets actifs d'un apprentissage ${ }^{7}$ qui à travers leur interaction et participation essaie d'en développer la personnalité et l'imagination ${ }^{8}$.

Les analogies de ces méthodes avec les positions innovantes proposées par l'italien Gianni Rodari sont étonnantes. En tant que théoricien de la jeunesse, il a repoussé la notion devenue tristement conventionnelle d'enseignement répressif (180) comprise comme apprivoisement, où des rôles figés établissent de façon très rigide la hiérarchie dans l'apprentissage. Il a dénoncé avec force l'urgence d'un renouvellement profond de l'école, dont il a soutenu sans cesse la force révolutionnaire. Pour ce faire, il est nécessaire que les enseignants se transforment en animateurs et qu'ils s'efforcent d'offrir les instruments pour que tous les enfants, selon leurs différentes habilités, puissent nourrir leur créativité (Rodari $178)^{9}$. Ce n'est que si ces conditions sont respectées que l'école remplira la fonction qui lui est propre, celle de développer l'esprit humain dans toutes les directions, et redeviendra ainsi le lieu privilégié pour l'enseignement de la liberté.

Un arrière-plan si riche a imposé une préparation à la traduction la plus transversale possible $^{10}$. On a commencé par s'intéresser au contexte africain et ivoirien, à l'histoire et à la culture, pour arriver ensuite à la production littéraire ${ }^{11}$. On s'est aussi arrêté sur les fondements du conte de fée, afin d'avoir une idée de la structure et de l'évolution que ce genre a subi pendant les siècles ${ }^{12}$. On a pu enfin préciser dans quelle mesure celui-ci peut être actualisé et réinventé, même transformé en instrument pour entraîner l’imagination des

\footnotetext{
${ }^{4}$ Michelle Tanon-Lora vit et travaille à Abidjan. Cette ville est affligée par tous les problèmes typiques d'une mégalopole africaine (criminalité, dispersion scolaire, pollution, dégradation de l'environnement). À ce propos (cf., Haeringer 27-40)

5 D'après les données de l'UNESCO, mises à jour en 2000, 51,4 \% de la population ivoirienne est analphabète, tandis que les dépenses publiques consacrées à l'éducation ont représenté 5\% du PIB en 1997 (dernières données disponibles).

${ }^{6}$ C'est à dire les activités dans lesquelles Michelle Tanon-Lora implique les enfants pendant les Ateliers des contes qui renvoient à la notion africaine d'art total (Rodari 178).

7 Pendant les Ateliers des contes documentés par Michelle Tanon-Lora, cette dernière utilise ses œuvres pour les finalités didactiques les plus différentes, de la sensibilisation aux problèmes de l'environnement à l'éducation à la participation.

8 Rodari consacre un long exposé à la reine des facultés, la puissance créatrice qui anime chacun de nous et qui est étouffée par la société bourgeoise. Cette dernière prétend l'attribuer à de rares d'élus pour pouvoir mieux contrôler tous les autres, en théorisant une différence qualitative entre les personnes.

9 Celle qui est à sa fois synonyme de "pensée divergente ", c'est-à-dire "capable de casser sans cesse les schémas de l'expérience, de découvrir des problèmes là où les autres trouvent des solutions satisfaisantes, de se sentir à l'aise là où les autres ne voient que des dangers, de formuler des jugements autonomes et indépendants (même par rapport au père, au professeur, à la société)» (Rodari 179).

10 On a cherché à ne pas «traduire pour comprendre » mais à «traduire après avoir compris » (Podeur Jeux 3).

11 On renvoie ici à la section bibliographique relative à la littérature africaine.

12 On renvoie ici à la section bibliographique relative aux œuvres de Vladimir Propp.
} 
enfants "non pas pour que tous soient des artistes, mais pour que personne ne soit esclave $^{13} \gg$ (Rodari 14).

Bien qu'Umberto Eco nie avec vigueur que Dire presque la même chose soit une œuvre de théorie de la traduction, elle a représenté le phare théorique de notre travail. En effet, elle s'est révélée fondamentale en ce qui concerne la prédisposition de l'esprit à la traduction et à la compréhension profonde du sens de cette activité. À travers cette œuvre, la traduction s'est révélée comme l'apogée d'un travail assidu d'appropriation du texte, suivi dans la plupart des cas par un échec inévitable parce que, sauf dans les rares cas de synonymie parfaite, " on ne dit jamais la même chose » (Eco 93). On est donc toujours obligé de choisir mot par mot, ce qu'il faut retenir et ce qu'il faut sacrifier, sans se réfugier dans les rangs confortables des «sourciers» et des «ciblistes» (Ladmiral 33-42) en prenant la responsabilité de choisir «chaque nuance» (111), sans rien laisser au gré du lecteur. Eco trace un modèle audacieux de fidélité au texte, qui se détache parfois complètement de la traduction littérale; il admet que le traducteur risque, dans le cas où, par exemple, il bouleverse la structure et la forme de phrases tout entières pour garder la nature profonde des macro-propositions (154-156).

Les deux textes de Podeur, Jeux de traduction et La pratica della traduzione ont servi de guide technique à l'usage des procédés traductifs directs et indirects, particulièrement dans les cas de transposition et de modulation :

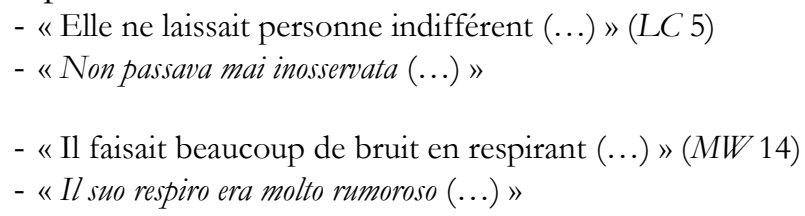

Dans le premier exemple, la perspective glisse des observateurs à l'objet de l'observation, tandis que dans le second on trouve deux changements de catégorie grammaticale (transposition en chaîne) (Podeur Jeux 40, 2008) qui transforment un couple de verbes (" faire du bruit» peut être vu comme une seule locution verbale) en un syntagme nominal.

Le nom Mamy Wata a demandé une adaptation, dont le résultat a été en italien Mama Acqua. Même si la connotation de respect (Zaourou 75) et de tendresse qui marque l'original français pour le lecteur anglophone et francophone (N’Da 141) est perdue, la solution proposée a été choisie pour sa capacité à rappeler à l'esprit du lecteur italien des échos familiers, comme par exemple le personnage de la domestique du célèbre Autant en emporte le vent, ou la chanson Mama Africa, écrite par Peter Tosh, qui donne son nom au recueil et qui est très connue dans l'interprétation de la chanteuse Sud-Africaine Miriam Makeba (surnommée à son tour Mama Africa ${ }^{14}$.

13 Il faut rappeler que d'après Rodari, le développement de l'esprit humain en toutes directions est le présupposé fondamental pour l'émancipation. Pour cette raison, la société productive vise à bloquer tout cela au nom de la fragmentation et de la spécialisation qui finissent par créer de « fidèles exécuteurs et de dociles reproducteurs ». L'éducation a en tout cas le pouvoir de changer la société, dans un sens ou dans l'autre.

${ }^{14}$ La chanteuse est décédée le dimanche 9 novembre 2008 à Castel Volturno, près de Naples, des suites d'un malaise. Elle venait de terminer un concert de soutien à l'écrivain italien Roberto Saviano. 
Au niveau de l'intrigue, il est très difficile de reconduire Masque, raconte-moi aux sept catégories de Paulme (19-50) vu qu'il ne s'agit pas d'une narration au sens propre du terme. Il s'agit plutôt d'un dialogue entre le Masque ${ }^{15}$ et l'enfant qui, pendant l'initiation, meurt symboliquement pour renaître homme, et obtient ainsi les connaissances nécessaires pour agir sur la nature et sur les animaux en particulier (ce qui est très important pour des sociétés primitives qui vivent de chasse ${ }^{16}$ ). Le rituel implique la fusion entre l'homme et l'animal (Propp 233-305) ${ }^{17}$, ce qui donnera au premier la possibilité d'exercer son pouvoir sur le second. La capacité de l'enfant de nommer cet animal en détermine la victoire (Paulme La mère 173).

De son côté, Mamy Wata correspond parfaitement au premier modèle morphologique (Ibid. 26) de conte africain, c'est-à-dire le «conte ascendant» qui commence par une situation initiale de manque pour arriver, après nombre de difficultés, à une amélioration. L'histoire est celle de Mamy Wata, reine de l'eau, aux prises avec un monstre terrible qui se nourrit d'êtres humains. Elle s'aperçoit que ce dernier n'est qu'un jeune homme victime d'une sorcellerie. Elle entreprend donc de lui redonner la joie. Mamy Wata arrive à le faire sourire, et ce sourire brise le maléfice. Le jeune homme décide enfin de vivre avec la reine de l'eau pour toujours. Les symboles sont ici puissants et les références nombreuses : le jour et la nuit, la recomposition de l'androgyne (cf., Chevalier \& Gheebran 39-41), la sorcière $^{18}$, ce monstre qui a beaucoup en commun avec le dragon de Propp affamé de vies humaines ${ }^{19}$, le pouvoir des mots, la capacité de l'art d'élever l'homme, le jeu considéré, avec

${ }^{15} \mathrm{La}$ valeur du Masque en Afrique est énorme et il serait impossible de l'approfondir ici. On se limitera à rappeler qu'il peut être le siège de forces surnaturelles, et qu'il exige donc des cultes très strictes dont la pratique est jalousement gardée par des sociétés secrètes masculines qui s'occupent aussi de l'initiation des enfants. (cf., Mossetto \& Rasch 261-283 ; Chevalier \& Gheebran 614-618).

${ }^{16}$ Dans une société où le progrès technologique se trouve encore au début, l'élément fondamental pour la chasse est la force magique, c'est-à-dire la capacité d'attirer la proie. Mais, «au fur et à mesure que les instruments se perfectionnent, [...] la force magique, précédemment attribuée à l'animal tutélaire, se retrouve ensuite dans l'instrument. Il en découle l'idée que l'instrument ne travaille pas parce qu'on lui a appliqué une force, (plus l'instrument est sophistiqué, moins d'effort est nécessaire) mais parce qu'il possède des capacités magiques indépendantes ». (Propp 210). [C'est nous qui traduisons]

17 Propp développe très longuement ce sujet. On pourrait tenter une synthèse simpliste en rappelant que quelques rites d'initiation prévoyaient l'entrée du néophyte dans une case, décorée pour ressembler à un animal, afin qu'il ait l'impression d'être englouti. Sans doute, une fois à l'intérieur, mangeait-il à son tour un bout du même animal, pour établir une relation d'appartenance ou d'identification avec celui-ci. Le fondement psychologique, qui remonte à la préhistoire, est celui de la co-essence avec ce que l'on mange. Il s'ensuit qu'un lien naît entre l'animal et son totem, c'est-à-dire son animal tutélaire (Chevalier \& Gheerbrandt 959).

${ }^{18}$ Dans laquelle on peut aisément reconnaitre la véritable antagoniste de Mamy Wata, sa projection renversée, ou la mère dévorante (cf., Paulme La mère dévorante 285-286).

${ }^{19} \mathrm{Il}$ y a aussi dans le texte beaucoup d'allusions aux rites d'initiation et à ceux de propitiation qui demandaient souvent des sacrifices humains, particulièrement de jeunes vierges ou d'enfants (considérés comme une garantie de pureté absolue). (cf., Propp 233-261). 
le rire, comme le propre de l'homme ${ }^{20}$. La rencontre avec l'autre et la méfiance envers les apparences, restent toutefois les thèmes principaux.

En ce qui concerne La ceinture de Madame fourmi, il s'agit d'un exemple de «conte descendant» (Paulme La mère 28) où l'état de manque arrive après des péripéties qui gâtent une situation initiale stable. La belle madame Fourmi doit aller à la fête de la récolte qui a lieu dans la capitale et demande à sa voisine la coccinelle de lui prêter sa belle ceinture en cuir rouge. Pour bien se faire remarquer partout dans le village, elle met la ceinture une semaine avant le début de la fête. Quand cette dernière arrive enfin, madame Fourmi renonce même à dormir, pour danser pendant des jours entiers. De retour chez elle, malgré l'insistance de la coccinelle, elle refuse de lui rendre la ceinture. Elle la garde des mois entiers, et ne l'enlève jamais, de peur que quelqu'un ne la lui prenne en cachette. Elle la ramène enfin à la coccinelle mais, comme la ceinture a été attachée à sa taille pendant trop longtemps, elle a rétréci, au point que la fourmi n'arrive plus à rester debout sans risquer de se couper en deux. C'est pour cette raison que depuis ce moment-là, la fourmi rase les murs et évite les lieux trop fréquentés. Dans une société tellement collectiviste, où la cohésion sociale est très forte et où « l'individu se définit par son statut et non d'abord pour ses qualités personnelles» (Paulme La mère 68), c'est la juste punition pour des manifestations de vanité et d'égocentrisme qui pourraient amener à la tentation de l'individualisme.

Quant à l'emploi de la langue, il y a bien sûr beaucoup de différences entre nos deux auteurs, mais il n'est pas très difficile d'envisager les analogies qui marquent leur travail. Même quand Véronique Tadjo s'adresse (formellement du moins, comme on l'a déjà vu) aux enfants, elle ne renonce pas à une prose fortement rythmée, structurée en vers libres, souvent brisés et riches en allitérations (que l'on relève plutôt dans Mamy Wata que dans Masque, raconte-mor). Le texte en sort lent et musical à la fois, bien scandé pour une lecture à haute voix, les mots vibrent en effet sur la page vide : "Il laissait échapper/ de longs gémissements/ et faisait beaucoup de bruit/ en respirant» ( $M W$ 15). Voilà pourquoi le texte a été traduit sur la base de nombreux enseignements théoriques concernant le respect de la musicalité du texte source ${ }^{21}$. Toutefois, les pertes dans le texte d'arrivée sont évidentes : «Emetteva/ lunghi gemiti/ e il suo respiro/ era molto rumoroso.»

Les grands espaces vides servent de caisse de résonance et ils représentent une caractéristique constante dans l'œuvre de Véronique Tadjo. Cependant, dans le cas de la littérature pour la jeunesse, ils ont une autre fonction importante : celle de porter l'attention du lecteur sur les images et les couleurs (Tsibinda 1,12); plus le public cible est jeune, plus les couleurs seront voyantes. Véronique Tadjo est bien consciente de l'effet que les

\footnotetext{
20 «L'homme ne joue qu'au moment où il est homme au sens plein du mot, et il n'est complètement homme que quand il joue », de Schiller (cité in Rodari 181). Voir aussi à ce propos l'essai de Johan Huizinga intitulé Homo ludens, essai sur la fonction sociale du jeu.

${ }^{21}$ «En poésie, c'est l'expression qui règle le contenu. [...] Verba tene, res sequentur» (Eco 56). D'après Eco, la fidélité au rythme du texte est fondamentale. Si ce dernier est décisif pour l'effet sur le lecteur, le bouleversement sémantique total est justifié, ce qui sacrifierait la « réversibilité » de la traduction, c'est-à-dire la possibilité de remonter au texte source en partant du texte cible (68-69).
} 
couleurs exercent sur les enfants, et elle les utilise comme un appât pour les attirer vers la lecture $^{22}$. Les illustrations (créées par Véronique Tadjo elle-même) ont donc ici une valeur fondamentale et sont chargées de significations symboliques: il suffit de penser à la recomposition de l'« œuf cosmique » (p. 9) ${ }^{23}$ ou à la «main tendue » (p. 7$)^{24}$ dans $M W$.

L'écriture de Michelle Tanon-Lora est bien sûr plus prosaïque, mais comme chez Véronique Tadjo, on y trouve aussi cette répétition typique de l'oralité :

- $\quad$ «Regarde-moi bien./Que vois-tu?/ Regarde-moi encore» (MR 5) ${ }^{25}$.

- $\quad$ "Cette ceinture te va à ravir» (LC 8-14).

En outre, on peut remarquer beaucoup de formules figées qui appartiennent depuis toujours au conte :

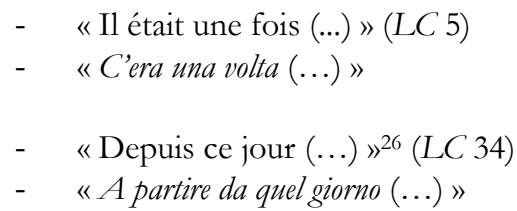

En italien, il s'est avéré nécessaire d'utiliser leurs correspondants traditionnels à travers un procédé de modulation.

Les deux écrivains utilisent un lexique essentiel qui engendre toutefois beaucoup de problèmes de traduction, sans doute à cause de sa simplicité. Véronique Tadjo préfère les mots particulièrement évocateurs et symboliques, qui trouvent un écho très vaste dans l'imaginaire africain. Le mot «sorcière », par exemple, offre au lecteur africain un éventail d'images beaucoup plus varié que l'italien «strega $»^{27}$. La même difficulté se présente avec une multitude d'autres mots, sans que la traduction arrive à faire passer (traducere) avec succès la grande partie de l'univers métalinguistique qui se cache derrière eux.

${ }^{22}$ Comme l'explique Véronique Tadjo : «Ils aiment les couleurs vives et les mouvements. Cela capte leur attention. Une fois qu'ils sont attirés par une illustration, ils ont envie de connaittre l'histoire qui va avec. Comme les enfants ont une grande imagination, ils aiment qu'on les stimule de ce côté-là. [...] Cela dépend du thème que j'aborde et de l'âge des enfants pour lesquels j'écris. Plus ils sont jeunes et plus les couleurs sont vives » (Koulibaly 7-8).

${ }^{23}$ En simplifiant, on pourrait le définir comme l'état d'unité parfaite avant la chute, le noyau originaire où les opposés coïncident (cf., Chevalier \& Gheerbrandt 693).

${ }^{24}$ Les interprétations possibles de cette illustration sont très nombreuses. Il faut toutefois remarquer que Véronique Tadjo souhaite qu'il y ait plus de dialogue entre Afrique francophone et anglophone. Le choix d'un sujet qui s'inspire d'un mythe diffusé dans les deux sphères linguistiques n'est sans aucun doute dû au hasard. ${ }^{25} \mathrm{Il}$ faut remarquer que dans un texte de 477 mots, cette séquence apparait 5 fois.

${ }^{26}$ Cette expression a le même effet que le «C'est pourquoi (...)» qui conclut généralement ce type de contes à propos de l'origine des éléments de la nature. (Cf. Mossetto \& Raschi 51).

${ }^{27}$ Il faut rappeler que Peter S. Thompson, dans sa note du traducteur de l'édition anglaise de Latérite, premier recueil de poèmes de l'auteur, explique la connotation fortement féminine de ce mot, très proche du domaine de la maternité. Ce n'est pas par hasard que la sorcière est aussi ici une mère qui punit le jeune homme pour avoir refusé d'épouser l'une de ses filles, en s'opposant ainsi de façon presque sacrilège à l'impératif de la procréation. Il faut remarquer qu'en Afrique le mariage est lié, avant qu'à la vie, à un sens de mort, à savoir celle de la famille d’origine des époux après le départ de ses enfants (cf., Paulme La mère 127). 
- «Animal » (MR 11), « chant» (MR 14), « mots » (MW 10), « eau » (MW 8), « caméléon » (MR 16), « source » (MR 11), « masque » (MR 2-23).»

- $\quad$ "Animale », « canto », "parola », « acqua », « camaleonte », «fonte », « maschera. »

Toutefois, l'hypothèse d'insérer des notes explicatives en bas de page a été tout de suite écartée pour deux raisons: d'une part, parce qu'on est conscient qu'elles doivent être l'ultima ratio pour le traducteur (Eco 95-96) et, d'autre part, par crainte de tomber involontairement dans une «explication»du texte ${ }^{28}$. C'est pourquoi, l'ambiguïté qui suit: «Petit oiseau chanceux/ qui connaît une grande liberté » (MR 14) a été traduite par l'adjectif «sconfinata», qui semblait bien résumer le sens profond de l'ambivalent « grand» (qui peut signifier littéralement "dont la taille est élevée » ou «dont les dimensions sont remarquables »), en cherchant à indiquer une liberté qui n'a pas de limites, capable de toucher le ciel qui est traditionnellement interdit aux hommes. Toutefois, la connotation de verticalité disparaît irrémédiablement.

De l'autre côté, même si les mots de Michelle Tanon-Lora apparaissent absolument univoques, tout le corpus lexical présente maintes difficultés. Les entropies les plus évidentes se vérifient toutefois pour les mots qui véhiculent la caractérisation locale: « marigot» (LC 9), «tam-tams », «balafons » (MR 9-10). "Marigot» n'a pas de correspondant direct en langue italienne. Dans le premier cas, en choisissant la traduction "pantano» selon le principe de négotiation (Eco 18), on a décidé de garder l'aspect de l'eau dormante, plus pertinent dans le texte en question. On n'a donc pas informé le lecteur qu'il s'agit d'une déviation d'un fleuve qui n'a pas de débouchés. Le mot français est une référence typique des régions équatoriales et cette connotation locale est, elle aussi, inévitablement perdue ${ }^{29}$.

En ce qui concerne « canaris », la perte la plus importante au niveau sémantique est bien sûr relative au matériel constitutif du récipient: la terre cuite. Le refus de la transcription et le choix d'« apprivoisement» (Eco 172) s'efforcent d'éviter toute tendance au pittoresque, si contesté dans l'histoire de la littérature africaine (Chevrier 17). En outre, la strophe dans laquelle ce terme apparaît est accompagnée par une illustration nécessaire pour compléter le sens des mots : en cela elle permet de compenser les pertes du texte d'arrivée. Le même raisonnement nous a conduit à la traduction de «tam-tams » par «tamburi », et à la perte de la forte connotation géographique qui en découle. Par contre, le terme "balafon» a été transcrit, car son correspondant italien «balafong», invariable, n'aurait pas éliminé l'éloignement que même le lecteur moyen francophone non africain ressent. Pour obtenir le même effet dans le texte cible (cf., Podeur La pratica 113), on a donc choisi de retranscrire la forme française qui ne provoque aucune distorsion du son conçu dans la version originelle par l'auteur. À la fin du texte, Michelle Tanon-Lora présente un véritable glossaire visant à l'explication des "mots difficiles", tandis que la note en bas de la page 6 de la

\footnotetext{
${ }^{28}$ La traduction ne doit rien expliquer, elle ne peut qu'expliciter les éléments qui sont déjà implicites dans la langue du texte de départ. Elle ne doit ni enrichir, ni améliorer le texte : si cela se produit, le résultat sera peutêtre un texte excellent, mais il ne sera point une bonne traduction (cf. Podeur Jeux 18 ; Eco 110).

${ }^{29}$ Le lecteur italien aura peut-être tendance à voir cet aspect dans une perspective un peu négative, à cause de l'usage figuré courant des termes «palude» et «pantano». Cette perte résultera plus grave encore si l'on considère que la réalité du marécage est liée de façon très positive à la réalité ivoirienne. Abidjan, le cœur économique et culturel du pays, se développe en effet sur une lagune, très proche de la mer, dont les Ivoiriens sont très fiers.
} 
Ceinture pour expliquer le "Titai», révèle l'intention d'établir un contact entre les nouvelles générations, notamment celles des métropoles africaines, et les rituels de la tradition.

Au niveau morphologique, aussi bien dans le cas de Mamy Wata que de la Ceinture, l'adjectif et l'adverbe sont utilisés avec sobriété, sauf dans le passage qui introduit madame Fourmi :

- $\quad$ «(...) Elle avait une belle peau lisse, des dents aussi blanches que des perles, et une chevelure soyeuse et onduleuse. » (LC 5).

- « (...) Aveva una bella pelle liscia, denti bianchi come perle e una chioma setosa e ondulata. »

Au contraire, Masque, raconte-moi est un texte descriptif, où les adjectifs sont très nombreux et placés au début de la phrase, en guise d'épithètes ou comme s'il s'agissait de nombreuses variations d'une même anaphore. En outre, on préfère la variation adverbiale ou l'adjectif à l'adverbe, afin d'éviter toute construction trop complexe qui ne reproduirait pas le style français:

- $\quad$ «Prudent, il pose délicatement une patte après l'autre (...)» (MR 16)

- "Accortamente, posa con delicatezza una zampa dopo l'altra (...)»

- « Riait aux éclats (...)» (MR 20)

- «Rideva fragorosamente (...)»

"Prudent» a ici une valeur adverbiale, tandis que l'adverbe qui se termine avec le suffixe «ment»a été choisi dans le texte sans doute pour des raisons phonétiques. Dans la Ceinture, on trouve une autre exception à cet usage (Podeur La pratica 46) :

- «Elle se déplaçait en avançant gracieusement (...)» (LC 5)

- «Incedeva con grazia (...)»

Les prépositions ne sont pas plus simples à résoudre :

- «Se couper en deux par la taille (...)» (LC 34)

- "Che la sua vita si spezzi in due (...)»

- «Avec ses boutoirs, ses verrues [...], il vaut mieux ne pas le croiser sur son chemin » (MR 13).

- "A giudicare da grugno, verruche [...], meglio non incontrarlo. »

La préposition "par» a demandé un véritable procédé de modulation, et dans le second cas on a eu recours à une transposition pour traduire « avec ».

En ce qui concerne l'usage des temps et des modes verbaux, l'imparfait domine en français, même là où l'italien préférerait d'autres solutions pour indiquer une action qui n'est pas habituelle ou pour mettre en évidence une action ponctuelle qui s'insère dans une action continue :

- «Trois jeunes filles, qui venaient remplir leurs canaris $(. .) ».(M W 9)$

- «Tre fanciulle, venute a riempire le brocche (...)»

- « Alors qu’il riait encore, il s’aperçut qu'il avait complètement changé. » (MW 20)

- "Mentre stava ancora ridendo, si accorse che era completamente cambiato." 
L'imparfait «venaient» a été remplacé par un participe passé, pour souligner le fait que l'action se déroule avant l'engloutissement. Les pages qui précèdent ce paragraphe nous expliquent que le monstre « guettait ses proies/ et se jetait sur elles/ lorsqu'elles approchaient de l'eau» ( $M W$ 8). On pourrait donc paraphraser ainsi l'action: trois jeunes filles vont remplir leurs canaris; une fois qu'elles se sont approchées de l'eau, elles sont englouties par le monstre. Entre leur arrivée et leur engloutissement, il y a une relation de conséquence et non pas de contemporanéité. C'est pourquoi, on s'est éloigné de la traduction littérale et on n'a pas utilisé un imparfait qui, en italien, aurait pu créer une certaine ambiguïté. Le même type d'intervention a été mise au point pour l'imparfait « riait». La structure italienne «stare + gerundio » offre une traduction plus précise là où un imparfait aurait été aussi possible; en outre, elle intensifie le contraste avec le passé simple «s'aperçut », «si accorse». Le gallicisme "être en train de» a sans doute été considéré par l'auteur comme une structure trop lourde qui aurait pu compromettre la fluidité du texte source.

Nous avons encore relevé plusieurs cas de nominalisation, là où l'italien ne dédaignerait pas la verbalisation, mais il s'agit ici d'un phénomène qui est désormais devenu assez fréquent dans la traduction du français en italien (Podeur La Practica 38) :

- «Depuis ton retour du Titai (...)» (LC 25)

- «Da quando sei tornata dal Titai (...)»

La plupart des textes présentent une structure paratactique, coordonnée par une asyndète. L'ordre des éléments syntactiques ne subit pas de bouleversements, même si l'italien permet parfois de trouver des solutions plus emphatiques, que la syntaxe française, plus rigide, n'accepterait pas :

- $\quad$ «a légende dit (...)» (MR 8)

- « Dice la leggenda (...)»

Une telle solution pourrait être un exemple d'« amélioration involontaire » (Eco 110) du texte source. En revanche, l'altération de l'ordre syntactique se révèle parfois indispensable :

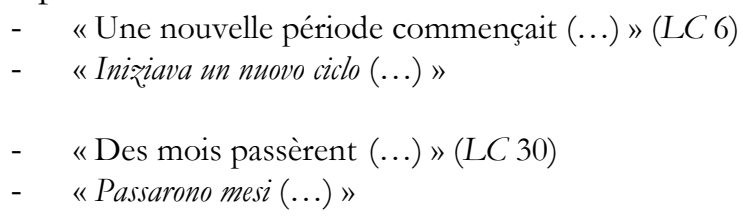

Nous pouvons aisément nous apercevoir du fait qu'une traduction littérale, qui présente donc la même structure syntaxique que le texte source, ne serait absolument pas naturelle pour le lecteur italien, plutôt habitué à une certaine fluidité linguistique.

On a cherché à présenter ici quelques-uns des éléments dont se compose le mélange indissoluble entre forme et sens à la base de chaque texte littéraire. La difficulté du traducteur consiste à déchiffrer ce réseau de reférences intra et metatextuelles et à les 
recréer dans sa propre langue : ce processus, qui met au jour les limites nécessaires de toute forme de communication, dénature en quelque mesure le texte, mais il lui offre aussi une nouvelle vie, celle des nouveaux lecteurs qui vont se l'approprier. 


\section{Bibliographie}

Boch, Raul. Il Boch, dirionario francese-italiano italiano-francese. Bologna: Zanichelli, 2008.

Chevalier Jean, Gheebrant Alain. Dictionnaire des symboles. Paris: Robert Laffont/Jupiter, 1982.

Chevrier, Jacques. Littérature nègre. Paris: Armand Colin, 1989.

De Mauro, Tullio. Il dirionario della lingua italiana. Torino: Paravia, 2000.

Eco, Umberto. Dire quasi la stessa cosa. Esperienze di tradurione. Milano: Bompiani, 2003.

Haeringer, Philippe. «Abidjan a cent ans. » Africultures, n 56, juillet-septembre 2003 : 2740.

Huizinga, Johan. Homo ludens, essai sur la fonction sociale du jeu. Paris: Gallimard, 1988.

Koulibaly, Isaïe Biton. "Véronique Tadjo vient de publier pour la jeunesse aux NEI 'Le Seigneur de la danse' et 'Mamy Wata et le Monstre'. » Amina, nº 287 (1994), p.7-8.

Ladmiral, J.-R. "Sourciers et ciblistes. » Revue d'Esthétique 12 (1986) : 33-42.

Mossetto Anna Paola, Raschi Nataša. Regards sur la littérature de Côte d'Ivoire. Roma: Bulzoni Editore, 1999.

N'Da, Pierre. "Les noms propres et les mots de la langue maternelle chez Maurice Bandaman". Francophonie littéraire et identités culturelles. Actes du colloque du Grelef (Cotonou, 1820 mars 1998). Ed. Adrien Huannou. Paris: L'Harmattan, 2000. 137-153.

Paulme, Denise. La mère dévorante. Essai sur la morphologie des contes africains. Paris: Gallimard, 1976.

---. Le civiltà africane. Milano: Garzanti, 1962.

Pellegrino, Isabella. Riflessioni sulla lingua di alcuni testi africani per l'infanzia. Sous la direction de Nataša Raschi. Perugia: n. p., 2010.

Podeur, Josiane. Jeux de traduction. Napoli: Liguori Editore, 2008.

---. La pratica della tradurione. Dal francese allitaliano e dallitaliano al francese. Napoli: Liguori Editore, 2002.

Propp, Vladimir. La morfologia della fiaba. Torino: Einaudi, 1966.

---. Le radici storiche dei racconti di magia. Trad. Salvatore Arcella. Roma: Newton Compton Editori, 1982.

Queneau, Raymond. Eserciri di stile. Introduzione di Umberto Eco. Torino: Einaudi, 1983.

Robert, Paul. Le nouveau Petit Robert de la langue française. Paris: Sejer, 2009.

Rodari, Gianni. La grammatica della fantasia. Torino: Einaudi, 2009.

Tadjo, Véronique. Mamy W ata et le monstre. London: Milet Publishing Ltd, 2000.

---. Masque, raconte-moi. Abidjan : NEI, 2002.

---. Red Earth - Latérite. (bilingual edition). Trans. Peter Thompson. Preface by Abiola Irele. Washington: Washington University Press, 2006.

Tanon-Lora, Michelle. La ceinture de madame Fourmi. Abidjan: Les Classiques ivoiriens, 2009.

Tsibinda, Marie-Léontine. "Véronique Tadjo : Poète, romancière et peintre.» Amina, $\mathrm{n}^{\circ}$ 472 (août 2009), pp. Europe 1 et 12.

Zadi Zaourou, Bernard. Césarienne. Abidjan: CEDA, 1984. 


\section{Sitographie}

Africultures association. Africultures. N. p., n. d. Web. 15 Mar. 2010.

Tadjo, Véronique. Véronique Tadjo. N. p., n. d. Web. 20 Mar. 2010.

Tanon-Lora, Michelle. Michelle Tanon-Lora. Afriblog, 2006. Web. 20 Apr. 2010.

Volet, Jean-Marie. Reading women writers and African literatures. Univ. of Western Australia, 1995. Web. 15 Apr. 2010. 